\title{
Cárdenas, Julián (2014): El poder económico mundial. Análisis de redes de interlocking directorates y variedades de capitalismo. Madrid: CIS, 283 pp. ISBN: 978-84-7476-639-4.
}

Durante los años 90 del pasado siglo se produjo toda una explosión de estudios sobre el fenómeno de la globalización. Algunos de las primeras plumas de la sociología: Giddens, Bauman o Beck, por citar apenas las más evidentes, dedicaron monografías a este proceso social. Hoy el debate no arrecia dentro de las ciencias sociales, pero parece haberse desplazado del mundo de la "gran teoría" al de los estudios empíricos sobre la globalización. El profesor Julián Cárdenas realiza un loable intento de describir cómo se estructura el poder de la economía global. Para ello, se centra en el análisis de las redes de interlocking directorates, es decir, analiza los consejeros delegados que tienen asiento en diferentes corporaciones. Con ello pretende analizar los vínculos que establecen las elites económicas corporativas como un medio para analizar el "poder económico mundial". Para ello, usa las herramientas metodológicas que ha desarrollado el análisis de redes sociales y el utillaje analítico de la "nueva sociología económica".

En la primera parte de la investigación se analiza el tipo de redes que surgen entre las 50 mayores corporaciones de 12 países: Países Bajos, Suecia, Italia, Francia, Alemania, España, Reino Unido, Estados Unidos, Suiza, Australia, Canadá y Japón. El profesor Cárdenas analiza tres dimensiones estructurales de estas redes: cohesión social, centralización o control por parte de pocos actores y multiplicidad, lo que indica que dos o más personas ocupan puestos en dos organizaciones. Estas dimensiones le sirven para establecer dos tipos de redes corporativas. En primer lugar, las redes elitistas que tendría una alta cohesión, una alta centralización y una alta multiplicidad. Los países en que predominan este tipo de redes son: Italia, Francia, Alemania, Canadá y España. En segundo lugar, las redes pluralistas caracterizadas por una baja cohesión social, una baja centralización y una baja multiplicidad. Los países que tienen este tipo de redes corporativas son: Japón, Reino Unido, Suiza, Estados Unidos, Australia, Países Bajón y Suecia.

En la segunda parte del estudio se tratan de hallar las causas que producen los dos tipos de redes de interlocking directorates descritos antes. Se parte de la idea de que las redes están insertas o incrustadas utiliza el concepto de embeddedness acuñado por Karl Polanyi- dentro de un entramado institucional y que las instituciones económicas propias de cada país determinarán el tipo de redes de interlocking directorates que aparezcan. Mantiene que existen cuatro grandes entramados institucionales y se elabora una tipología dicotómica de cada uno de ellos: estructura financiera, intervención del Estado en la economía, estructura de la propiedad y nivel de internacionalización. Plantea a modo de hipótesis que las redes pluralistas surgirán de un entorno institucional basado en bancos, con Estados intervencionistas, con la propiedad controlada por grandes accionistas y con una baja internacionalización. Por el contrario, las redes pluralistas surgirán de entornos institucionales basados en mercados de capitales, con Estados regulativos, con una propiedad dispersa y con alta internacionalización (Cuadro 1).

Cuadro 1. Entorno institucional de las redes corporativas y su relación con el tipo de red corporativa.

\begin{tabular}{lcc}
\hline \multicolumn{1}{c}{ Institución } & \multicolumn{2}{c}{ Tipología } \\
\hline Estructura financiera & Basada en bancos & $\begin{array}{c}\text { Basada en } \\
\text { mercados de } \\
\text { capitales }\end{array}$ \\
\hline $\begin{array}{l}\text { Intervención } \\
\text { del Estado }\end{array}$ & $\begin{array}{c}\text { Estados } \\
\text { intervencionistas }\end{array}$ & $\begin{array}{c}\text { Estados } \\
\text { regulativos }\end{array}$ \\
\hline $\begin{array}{l}\text { Estructura de la } \\
\text { propiedad }\end{array}$ & $\begin{array}{c}\text { Controlada por } \\
\text { grandes accionistas }\end{array}$ & $\begin{array}{c}\text { Propiedad } \\
\text { dispersa }\end{array}$ \\
\hline $\begin{array}{l}\text { Internacionalización } \\
\text { Baja }\end{array}$ & Alta \\
\hline $\begin{array}{l}\text { Tipo de red } \\
\text { corporativa }\end{array}$ & Elitista & Pluralista \\
\hline Fuente: Elaboración propia a partir de Cárdenas, 2014: 82-113.
\end{tabular}

Posteriormente, trata de comprobar estas hipótesis utilizando el análisis comparativo de carácter cualitativo y los fuzzy sets (fsQCA) tomados de la obra de Charles Ragin. El análisis muestra que "las redes corporativas elitistas están determinadas por la conjunción de la estructura financiera basada en los bancos, el estado intervencionista y las corporaciones controladas por los grandes accionistas y la baja internacionalización" (p. 133). De los casos estudiados, se encuadrarían aquí Italia, Francia, Alemania y España. Por el contrario, la estructura financiera basada en mercados de capitales, un Estado regulativo y la propiedad dispersa explicarían la emergencia de redes corporativas de tipo pluralista. Esto se produce en Estados Unidos, Reino Unido, Suiza, Australia y Canadá -aunque este último país es peculiar ya que su red tiende al elitismo pese a que institucionalmente debería ser pluralista-. El caso de Suecia y los Países Bajos se explicaría por una combinación de Estado regulativo, control por parte de los grades accionistas y alta internacionalización. La red japonesa, por último, no es explicada por este modelo, porque es "totalmente específica". 
El estudio concluye con un tercer bloque en el cual se analiza la existencia de diferentes variedades de capitalismo (VDC) y de una red corporativa mundial. En primer lugar, se plantea que lo expuesto sugiere la existencia de diferentes tipos de capitalismo en función de su organización institucional. Tras revisar las diferentes tipologías existente, plantea una propia organizada en torno a cinco ejes institucionales: tipo de red corporativa, nivel de internacionalización, estructura financiera, estructura de la propiedad e intervención del Estado. Tras realizar un análisis de conglomerados obtiene cuatro variedades de capitalismo: capitalismo basado en redes, capitalismo de mercado, capitalismo intermedio y capitalismo nipón (Cuadro 2).

Las explicaciones que se dan a la existencia de VDC han seguido dos ejes. El primero señala que las VDC son producto de las condiciones de inicio (path dependence) en las que se implantó el capitalismo. Y el segundo incide en lo que denominan complementariedades institucionales que hacen que las diferentes instituciones se refuercen mutuamente $y$ mantengan una determinada variedad de capitalismo. El autor considera que estas dos opciones tienen carencias que deben ser complementadas utilizando la variable poder, tal como hace la teoría de redes, En este sentido, habla de una jerarquía institucional, por la cual alguna de las instituciones presente en cada VDC resulta central al sistema. La institución en la cúspide sería la más importante para la clase dominante. Considera que de las analizadas el sistema financiero ocupa un lugar central.

Posteriormente el autor abandona el análisis de la estructura de la red corporativa a nivel nacional y pasa a realizar un análisis de la red corporativa a nivel global. Con ello, sin duda, intenta superar el nacionalismo metodológico que abunda en buena parte de los estudios empíricos sobre el fenómeno de la globalización. Para ello analizan las relaciones de interlocking directorates de las 338 corporaciones más grandes del planeta. De estas el $49 \%$ son de Estados Unidos, el 29\% de la Unión Europea y el 7\% de Japón, es decir, el $85 \%$ de las mismas proviene de estos tres entornos geográficos. Resulta significativo que en la red corporativa mundial está muy cohesionada, pues un $85 \%$ de las empresas están conectadas (p. 196). El análisis muestra algunas características destacadas. Así, las corporaciones japonesas aparecen como las más desvinculadas de la red. El resto aparecen agrupadas en torno dos grandes ejes. Por un lado, las empresas de la órbita anglosajona (Estados Unidos, Reino Unido,
Suiza y los Países Bajos) y, por otro, las del eje francoalemán (Alemania, Francia, España, Italia y Canadá).

El análisis concluye preguntándose si se está implantando un capitalismo global único. Cárdenas piensa que existe un conflicto entre las diferentes VDC y que las elites de cada bloque capitalista tratan de imponer su visión para crear un capitalismo global que se amolde a sus intereses. El uso de interlocking directorates sería una estrategia, entre otras, de las corporaciones para imponer su modelo de capitalismo global. Julían Cárdenas piensa que compartir lazos corporativos a través de los consejeros delegados conduce a que se construyan VDC semejantes. Significativamente, plantea que dentro de la Unión Europea la red corporativa se articula en torno al eje franco-alemán con una importante presencia de empresas del Reino Unido. Respecto al futuro, piensa que se mantendrán las VDC hasta que la institución rectora: el sistema financiero, no se unifique. Mientras se mantenga la financiación basada en bancos permanecerán las diferencias, cuando se vuelquen hacia los mercados se producirá una homogenización del capitalismo hacia un capitalismo de mercado.

En definitiva, resulta un estudio de enorme interés y una aproximación de obligada lectura para comprender como se estructura el poder en la actual economía globalizada. Valga como ejemplo postrero la imagen que se da de las redes corporativas en nuestro país. Estas se caracterizarían por ser del tipo elitista, es decir, cohesionadas, controladas por pocos actores centrales y con alta multiplicidad. Este tipo de red sería producida por una economía basada en una estructura financiera bancaria, controlada por grandes accionistas, con un Estado altamente intervencionista y con una baja internacionalización. Nos encuadraríamos claramente dentro del la variedad de capitalismo basado en redes. A esto se sumaría que la inserción en la red corporativa mundial de las corporaciones españolas estaría dentro de la Europa continental comandada por el eje franco-alemán. Una imagen especular de nuestro país que, sin duda, ayuda a entender la situación actual.

Antonio Martín Cabello Universidad Rey Juan Carlos, Madrid, España antonio.martin@urjc.es Recibida: 15-07-2014 Aceptada: 03-09-2014

\begin{tabular}{|c|c|c|c|c|c|c|}
\hline $\begin{array}{c}\text { VDC/Ejes } \\
\text { institucionales }\end{array}$ & $\begin{array}{l}\text { Tipo de red } \\
\text { corporativa }\end{array}$ & Nivel de internacionalización & $\begin{array}{l}\text { Estructura } \\
\text { financiera }\end{array}$ & $\begin{array}{c}\text { Estructura de la } \\
\text { propiedad }\end{array}$ & Intervención del Estado & Países \\
\hline Capitalismo de redes & Elitista & Media & $\begin{array}{c}\text { Basada en } \\
\text { bancos }\end{array}$ & $\begin{array}{c}\text { Centrada en } \\
\text { grandes accionistas }\end{array}$ & Intervencionista & $\begin{array}{c}\text { Alemania, España, } \\
\text { Francia e Italia }\end{array}$ \\
\hline $\begin{array}{l}\text { Capitalismo de } \\
\text { mercado }\end{array}$ & Pluralista & Alta & $\begin{array}{l}\text { Basada en } \\
\text { mercados } \\
\text { financieros }\end{array}$ & Dispersa & Regulativo & $\begin{array}{c}\text { Reino Unido, } \\
\text { Estados Unidos, } \\
\text { Australia y Suiza } \\
\end{array}$ \\
\hline $\begin{array}{l}\text { Capitalismo } \\
\text { intermedio }\end{array}$ & $\begin{array}{l}\text { Entre elitista y } \\
\text { pluralista }\end{array}$ & Alta & $\begin{array}{l}\text { Basada en } \\
\text { mercados } \\
\text { financieros }\end{array}$ & $\begin{array}{l}\text { Centrada en } \\
\text { grandes accionistas }\end{array}$ & $\begin{array}{c}\text { Regulativo, con políticas } \\
\text { intervencionistas en el } \\
\text { mercado exterior }\end{array}$ & $\begin{array}{l}\text { Países Bajos, } \\
\text { Canadá y Suecia }\end{array}$ \\
\hline Capitalismo nipón & Pluralista & $\begin{array}{c}\text { Baja (en relación a la inversión } \\
\text { extranjera directa) }\end{array}$ & $\begin{array}{c}\text { Basada en } \\
\text { bancos }\end{array}$ & $\begin{array}{l}\text { Un nivel medio de } \\
\text { grandes accionistas }\end{array}$ & Intervencionista & Japón \\
\hline
\end{tabular}

